\title{
Revamping Residency Education during a Pandemic with Twitter-Based Learning
}

\author{
Davis N. Bradford ${ }^{1} \cdot$ Robert M. Wilechansky $^{1}$ (D) $\cdot$ Alexandra R. Pipilas ${ }^{1} \cdot$ Kate M. Raiti-Palazzolo $^{1} \cdot$ Jason A. Sherer $^{1}$
}

Accepted: 23 November 2020 / Published online: 7 January 2021

(C) International Association of Medical Science Educators 2021

\begin{abstract}
Amidst the increasing clinical demands and social distancing constraints of COVID-19, Twitter-based, resident-driven education offers adaptability for the current predicament faced by residency programs and sparks curiosity that will outlast this pandemic.
\end{abstract}

Keywords Twitter $\cdot$ E-learning $\cdot$ Clinical education $\cdot$ Self-directed learning $\cdot$ Graduate medical education

With medical residents on the front lines of COVID-19 clinical care, maintaining emphasis on education can pose a challenge. Residents remain trainees first and foremost, and a continued focus on education is critical for their clinical growth, professional development, and morale. With social distancing measures rendering in-person morning reports and noon conferences unfeasible, we leveraged social media to not only preserve but also revamp education for the COVID-19 era.

In response to the drastic reduction in resident involvement in ambulatory settings, we created a Twitter-based, residentled education system entitled, "Five Magical Minutes," in which residents on ambulatory rotations generated clinical questions to post on the Boston University Internal Medicine Residency Twitter account (@BMCimRES) for inpatient teams to answer. Chief residents subsequently posted one clinical question daily from the repository created by residents on their ambulatory blocks. Question types varied from multiplechoice to open response - often with a hypothetical clinical scenario. Twitter's poll feature, which enables users to vote on the answer in real time, generated meaningful discussion among residents and the medical social media community. Answers to clinical questions were posted within $24 \mathrm{~h}$ and incorporated supporting evidence in a "tweetorial" format [1, 2], using sequential responses to the original post with links to relevant literature. Over a 10-week period when COVID-19

Robert M. Wilechansky

rmwilechansky@gmail.com

1 Evans Department of Medicine, Boston University School of Medicine, 72 East Concord St. Evans 124, Boston, MA 02118, USA was at its worst in our hospital, residents inspired 44 clinical questions in over 130 tweets, with each tweet averaging over 40 engagements (i.e., active interactions with the tweet) and over 1000 impressions (i.e., appearance on Twitter feed or searches).

This experience demonstrated how e-learning and social media can be meaningfully integrated into residency education. Resident-led Twitter education has empowered residents to "return to why," [1] fostering curiosity while respecting the constraints of busy clinical rotations. The self-directed, active learning strategy [3] employed in our initiative encourages residents to dig deeply into the primary literature and disseminate their knowledge to colleagues; developing these skills is a key step toward a career path in academic medicine and independent practice. By further harnessing the growing recognition of Med Twitter, residents engage with a broader audience, including trainees and faculty within and outside their institution.

As this pandemic has forced us to rethink health care, so too has social distancing forced us to reimagine resident education. While e-learning strategies may be unable to fully replicate in-person teaching, they serve as a complementary adjunct to modern medical education applicable across a wide spectrum of medical training programs. In the COVID era, resident-driven learning on a social media platform has allowed us to maintain the core of residency education, expand our reach, and develop innovations to last beyond the pandemic.

Acknowledgments The authors thank Gopala K. Yadavalli, MD, Internal Medicine Residency Program Director and Vice Chair for Education, Evans Department of Medicine, Boston University School of Medicine. 
Authors' Contributions Davis N. Bradford: concept, manuscript preparation, and critical revisions.

Robert M. Wilechansky: concept, manuscript preparation, and critical revisions.

Alexandra R. Pipilas: concept and critical revisions.

Katie M. Raiti-Palazzolo: concept and critical revisions.

Jason A. Sherer: concept and critical revisions.

\section{Compliance with Ethical Standards}

Conflict of Interest The authors declare that they have no conflict of interest.

Ethical Approval This editorial does not involve human subject research; approval was not obtained.

Consent As above, this editorial does not involve human subject research; consent is not applicable.

\section{References}

1. Breu AC. Why is a cow? Curiosity, tweetorials, and the return to why. N Engl J Med. 2019;381:1097-8.

2. Tony Breu on Twitter: " $1 /$ How does one create a Tweetorial? This is thread TWO of a THREE PART series. II. How to draft and post a Tweetorial. https://t.co/TvqcguZYoT" / Twitter. https://twitter.com/ tony_breu/status/1224015310619127809. Accessed 7 May 2020.

3. Lewis KO, Cidon MJ, Seto TL, Chen H, Mahan JD. Leveraging elearning in medical education. Curr Probl Pediatr Adolesc Health Care. 2014;44:150-63. https://doi.org/10.1016/j.cppeds.2014.01. 004 .

Publisher's Note Springer Nature remains neutral with regard to jurisdictional claims in published maps and institutional affiliations. 